ESPAÇO TEMÁTICO: PROTEÇÃO SOCIAL NO CAPITALISMO CONTEMPORÂNEO:

CONTRARREFORMAS E REGRESSÕES DOS DIREITOS SOCIAIS

\title{
Trabajo, derechos sociales y protección social en Argentina de la reconstrucción neoliberal
}

\author{
Malena Victoria Hopp ${ }^{1,2,3}$ \\ Eliana Lijterman ${ }^{1,2}$ \\ https://orcid.org/0000-0002-0532-0164 \\ https://orcid.org/0000-0002-5679-1919 \\ ${ }^{1}$ Universidad de Buenos Aires, Facultad de Ciencias Sociales, Instituto de Investigaciones Gino Germani, Ciudad Autónoma de Buenos \\ Aires, Argentina \\ ${ }^{2}$ Universidad de Buenos Aires, Facultad de Ciencias Sociales, Carrera de Trabajo Social, Ciudad Autónoma de Buenos Aires, Argentina \\ ${ }^{3}$ Centro Cultural de Cooperación Floreal Gorini, Ciudad Autónoma de Buenos Aires, Argentina
}

Trabajo, derechos sociales y protección social en Argentina de la reconstrucción neoliberal

Resumen: El artículo analiza la reorganización de la protección social en Argentina, iniciada a fines de 2015. Toma como analizadores la clausura de políticas centrales de la estrategia de inclusión por el trabajo que caracterizó al ciclo político previo: la moratoria previsional y la promoción de cooperativas de trabajo. En base al análisis documental se examinan los modos en que se reconstruyeron los sentidos del trabajo y el reconocimiento de derechos en la definición de los problemas sociales y las estrategias de intervención estatal para abordarlos. El análisis realizado muestra que los procesos de contrarreforma neoliberal en curso confluyen en una redefinición del valor social del trabajo y el horizonte de integración social y económica de las poblaciones vulnerables. Estos nuevos sentidos parten de una interpretación restringida y mercantilizada de los derechos sociales y del trabajo que tienen como efectos la asistencialización e individualización de las protecciones sociales.

Palabras clave: Seguridad social. Asistencia. Trabajo. Estado. Política social.

\section{Trabalho, direitos sociais e proteção social na Argentina da reconstrução neoliberal}

Resumo: O artigo analisa a reorganização da proteção social na Argentina iniciada no final de 2015. Toma como analisadores o encerramento de políticas centrais da estratégia de inclusão pelo trabalho que caracterizou o ciclo político anterior: a moratória previdenciária e a promoção das cooperativas de trabalho. Com base na análise documental, são examinadas as formas pelas quais se reconstruíram os significados do trabalho e o reconhecimento de direitos na definição dos problemas sociais e das estratégias de intervenção do Estado para abordá-los. A análise realizada mostra que os processos de contrarreforma neoliberal em curso convergem na redefinição do valor social do trabalho e do horizonte de integração social e econômica das populações vulneráveis. Esses novos significados baseiam-se em uma interpretação restrita e mercantilizada dos direitos sociais e do trabalho, cujos efeitos são a assistencialização e a individualização das proteções sociais.

Palavras-chave: Seguridade social. Assistência. Trabalho. Estado. Política social.

Labor, social rights and social protection in Argentina based on the neoliberal reconstruction

Abstract: The article analyzes the reorganization of social protection in Argentina, which began at the end of 2015. The parameter of analysis is the end of central policies based on the strategy of inclusion through employment, which was a mark of the previous political cycle that declared a social security moratorium and promoted labor cooperatives. The study used documentary analysis and observed how the meaning of work was rebuilt, as well as looking at the recognition of rights in the definition of social problems, and the state's intervention strategies to address these problems. The analysis shows that the processes of neoliberal counter-reform in progress converge in the redefinition of the social value of work and the future of vulnerable populations' social and economic integration. These new meanings are based on a restricted and mercantile interpretation of social and labor rights, bringing as consequences the "assistencialization" and the individualization of social protection.

Keywords: Social security. Assistance. Labor. State. Social policy.

Recibido en 31.05.2018. Aprobado en 18.09.2018. Revisado en 28.01.2019

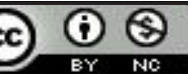

C El(Los) Autor(es). 2019 Acceso Abierto Esta obra está licenciada bajo los términos de la Licencia Creative Commons Atribución-NoComercial 4.0 Internacional (https:/creativecommons.org/licenses/by-nc/4.0/deed.es), 
que permite copiar, distribuir y reproducir en cualquier medio, así como también adaptar, transformar y crear a partir de este material, desde que para fines no comerciales, y que usted fornezca el crédito debido a los autores y a la fuente, insiera un enlace para la Licencia Creative Commons e indique si fueron hechas alteraciones. 


\section{Introducción}

El presente artículo analiza los procesos de reorganización de la protección social en Argentina, iniciados afines de 2015. En las elecciones de ese año, se impuso la Alianza Cambiemos y asumió la presidencia de la Nación Mauricio Macri, clausurándose, así, el ciclo político de administraciones kirchneristas. Por primera vez en la historia del país, accedió al poder por medios democráticos una alianza de sectores de centroderecha, que unifica la elite política y la económica. Ello representa una particularidad del caso argentino en comparación con otros procesos de descomposición y recomposición de los regímenes políticos en el proceso de reconstrucción neoliberal que experimenta la región latinoamericana.

Desde nuestra perspectiva, el neoliberalismo no conforma un ciclo de gobierno o de políticas, sino un proyecto político-cultural, que entraña formas de expresión y percepción del mundo social. Se trata de un régimen de existencia de lo social, quedebe ser comprendido en su capacidad de producción de formas de vida (Dardot \& Laval, 2013; Gago, 2014). Según la correlación de fuerzas sociales y políticas, el carácter neoliberal de las intervenciones sociales del Estado puede desplegarse con mayores o menores resistencias, marcando límites o habilitando posiciones reivindicativas y transformadoras de la protección y de las formas de integración social que ésta supone. Las reformas sociales se presentan como proyectos multiformes que tienen lugar de manera procesual, con marchas y contramarchas, a través de controversias y ejes de consenso entre fuerzas políticas heterogéneas.

El período 2003-2015, correspondiente al gobierno de Néstor Kirchner (2003-2007) y los dos mandatos de Cristina Fernández de Kirchner (2007-2015), se caracterizó por la centralidad asignada al trabajo regulado y protegido como vector de la integración social. Esto movilizó una serie de reorganizaciones de la política pública y, en particular, de la asistencia y la seguridad social. El actual gobierno de la Alianza Cambiemos fundamenta y disputa la legitimidad del modelo estatal en ciernes estableciendo una polémica con los rasgos definitorios del kirchnerismo. En esta confrontación, los fundamentos de la protección social como actividad estatal fueron puestos en cuestión y están siendo rediseñados. El problema que abordaremos aquí refiere a los modos de redefinición del valor social del trabajo como fundamento de las políticas destinadas a la protección social de grupos definidos como vulnerables por su débil e informal vinculación con el mercado de trabajo. Proponemos como analizadores de este proceso la clausura de dos políticas de amplio alcance y visibilidad, instituidas durante el kirchnerismo y las modalidades de intervención que las sucedieron:

A) El Plan de Inclusión Previsional, implementado por la Administración Nacional de la Seguridad Social (ANSES) en el año 2005, con una segunda etapa en 2014, cuyo objetivo fue garantizar el acceso a la jubilación a las personas que contaran con la edad pero no con los años de aportes suficientes para acceder al derecho, a través de un plan de regularización de la presunta deuda contraída conocido como Moratoria Previsional. El principio contributivo de la Seguridad Social fue reinterpretado, marcando un quiebre en su desarrollo histórico. Durante los años 2016 y 2017 se suscitaron dos reformas del sistema previsional que tendieron a una reversión de la moratoria, además de introducir otros cambios de relevancia que se encuentran en proceso.

B) Los programas de generación y apoyo a cooperativas de trabajo, Ingreso Social con Trabajo Argentina Trabaja, puesto en marcha en 2009 desde el Ministerio de Desarrollo Social de la Nación (MDS) y la línea Ellas Hacen, iniciada en 2013, destinada a mujeres con niños a cargo o víctimas de violencia de género. Estos habían tensionado las lógicas clásicas del campo asistencial y de la racionalidad neoliberal, basadas en la focalización a partir del principio de la necesidad, fundadas en la definición de los destinatarios por atributos personales, carencias o características específicas de su situación de pobreza. Ambos fueron eliminados en febrero de 2018 y reemplazados por el Programa Hacemos Futuro, una transferencia condicionada de ingresos de carácter personal, orientada a la formación laboral.

Los analizadores seleccionados permitirán poner en diálogo las formas de (re)definición de los problemas a los que se dirigen las políticas, las estrategias de abordaje, la consideración del trabajo y los modos de reconocimiento social que proponen. Puntualizaremosen los principios y fundamentos que orientan las intervenciones dirigidas a revertir las políticas del ciclo político anterior en el proceso de reconstrucción neoliberal en curso. El análisis se basa en una metodología cualitativa, sostenida en el trabajo de archivo, mediante el cual reconstruimos los discursos oficiales que fundamentan la redefinición del rol del trabajo en la política pública y de las intervenciones sociales en estudio. El corpus documental comprendió normativas, instructivos para la implementación de programas, sitios web oficiales, informes de gestión, documentos e información de prensa que recupera las manifestaciones públicas de funcionarios de gobierno.

El esquema de organización del artículo es el siguiente. Primero, desarrollamos las coordenadas conceptuales e históricas que guían el análisis sobre las transformaciones recientes dela protección y la asistencia social en Argentina. Luego analizamos las reformas en el campo de la previsión social y el modo en que éstas redefinen el trabajo como fundamento de la protección social. En tercer lugar examinamos el proceso de eliminación delas 
cooperativas de trabajo como núcleo de los programas de inclusión social de grupos en situación de vulnerabilidad, desempleo y pobreza y el pasaje a una estrategia de intervención basada en transferencias de ingresos condicionadas. En el último apartado, presentamos las conclusiones del análisis realizado.

\section{Asistencia y seguridad social: itinerarios en la historia argentina reciente}

En el desarrollo histórico de la política social, la oposición entre trabajo y pobreza ha sustentado un esquema de clasificación y organización institucional entre asistencia a los pobres y seguridad social a los trabajadores (Castel, 1997; Morell, 2002), que en Argentina asumió ciertas particularidades. Hasta los años 1980, los derechos sociales se expandieron a partir de la amplitud que adquirió la categoría de trabajador asalariado, sobre la cual se construyeron seguros sociales corporativos de amplia cobertura, aunque segmentada. La asistencia, en cambio, se ocupó de quienes quedaron en los márgenes del empleo, asumiendo un lugar residual en el esquema de política social (Soldano \& Andrenacci, 2005). La política social delimitó un campo de intensa transformación en el proceso de consolidación de la experiencia neoliberal argentina, cuyo eje fue la desocialización del trabajo y del bienestar (Danani, 2017b).

La connotación negativa que asumieron las ideas de protección y seguridad, condujeron a un proceso sin precedentes de individualización y mercantilización de la seguridad social (Cortés \& Marshall, 1999; Danani \& Lindenboim, 2003). La estrategia de política social reforzó su contenido asistencial al disociar la pobreza de las transformaciones del mundo del trabajo (Grassi, 2004). Durante los años 1990 se produjo una modificación del lugar residual que la asistencia social había ocupado hasta entonces y pasó a cobrar centralidad en el campo de las políticas sociales. El trabajo continuó perfilando el ideal del autosostenimiento y autonomía, aunque desprovisto de soportes sociales e institucionales, se volvió un mandato impuesto a todos, independientemente de su cualidad (Fraser, 1997, pp. 163-200).

La crisis económica, social y política que atravesó la Argentina en los años 2001 y 2002, marcó un resquebrajamiento de la hegemonía neoliberal y el cierre de un ciclo de políticas ${ }^{1}$. Luego de la caída del régimen de convertibilidad cambiaria, la asunción de Néstor Kirchner en la presidencia de la Nación en 2003 profundizó la reestructuración de las políticas económicas, laborales y sociales. Su legitimidad se fundó en la confrontación con el ciclo previo, definido como neoliberal. El modelo de Estado propuesto se basó en la expectativa de compatibilizar el crecimiento económico con la inclusión social, entendida en el discurso ofícial como la integración a partir del trabajo formal, regular y protegido. Éste se invistió de valor social y moral, al definirse como el factor productivo y distributivo por antonomasia y como organizador de la vida en sociedad y de la propia identidad (Comité Nacional MOST, 2015; Kirchner, 2007; Ministerio de Desarrollo Social, 2010). Las políticas laborales conformaron un campo estratégico de reformulación de las intervenciones sociales ${ }^{2}$ y la seguridad social se presentó como el sistema más virtuoso para organizar las protecciones, definiéndose como su núcleo (Golbert, 2010, pp. 9-11; Ministerio de Trabajo, Empleo y Seguridad Social, 2014).

En el campo de la seguridad social se desplegó un proceso material y político-cultural de inversión de la dirección de las reformas de los años noventa (Danani, 2017a; Danani \& Grassi, 2008). Caracterizó a este proceso el restablecimiento del carácter público y solidario del sistema y la fuerte ampliación de la cobertura con la incorporación de trabajadores informales y desocupados, históricamente excluidos de la seguridad social. Los mecanismos para dicha ampliación fueron heterogéneos: regímenes especiales para la formalización laboral; modalidades de reinterpretación del principio contributivo (como en el caso de la Moratoria Previsional); y el fortalecimiento y la creación de componentes no contributivos (del que la Asignación Universal por $\mathrm{Hijo}^{3}$ es un caso paradigmático). El alcance de estas medidas fue muy amplio ${ }^{4}$ y el reconocimiento hacia los trabajadores excluidos del empleo formal marcó un quiebre en la Seguridad Social argentina, alterando el sujeto que ésta había reconocido como propio y tensionando la relación históricamente establecida con el campo asistencial.

En consonancia con la definición del trabajo como eje central de la integración social, desde el MDS se impulsó un plan de generación y fortalecimiento del trabajo asociativo y cooperativo destinado a grupos en situación de vulnerabilidad, pobreza y/o desocupación (Resolución n. 3182, 2009). Esta línea programática intentó distinguirse de los planes asistenciales dela década previa, por un lado, a partir de concebir el ingreso que brindaba a los destinatarios como una retribución por el trabajo realizado en cooperativas. Por otro, mediante la búsqueda del fortalecimiento de nuevas organizaciones de trabajadores, como parte de una direccionalidad política e institucional hacia la conformación de espacios colectivos de trabajo orientados por valores de solidaridad, inclusión y compromiso con la comunidad (Hopp, 2017b).

La Alianza Cambiemos, que se impuso en las elecciones presidenciales del año 2015, se conformó a partir de una reorganización de partidos de oposición al kirchnerismo ${ }^{5}$.Su discurso conectó con ciertas expresiones de descontento social entre capas medias y bajas (Rofman \& Zamora, n.d.), entablando una confrontación 
directa con las orientaciones de las intervenciones estatales del ciclo previo. Bajo el argumento de que el Estado debe favorecer el libre desarrollo de la actividad privada para reducir el déficit fiscal y expandir el bienestar de manera genuina, se avanzó en profundas transformaciones de la política económica: una fuerte devaluación de la moneda, reducción impositiva a importaciones, eliminación de retenciones a la exportación de productos agrícolas, quita de subsidios a servicios públicos, entre otras. Estas medidas tendieron a fortalecer a los sectores agroexportador, financiero y a empresas de capital concentrado, en desmedro de las pequeñas y medianas empresas y de la industria local. En consecuencia, se alteró la composición del empleo, cuyo crecimiento fue liderado porlos segmentos de mayor incidencia de la informalidad y de menores ingresos (Instituto Nacional de Estadística y Censos, 2018; Ministerio de Trabajo, Empleo y Seguridad Social, 2018). La creciente inflación y el cambio de orientación gubernamental en las negociaciones salariales generaron un deterioro continuo del poder adquisitivo (Centro de Estudios Económicos y Sociales Scalabrini Ortiz, 2016).

A partir de 2017, el oficialismo impulsó el debate de una triple reforma: previsional, tributaria y laboral. Los ritmos de los procesos de negociación política condicionaron a que ésta última aún no haya tenido tratamiento, mientras que la previsional fue sancionada en el marco de una intensa conflictividad social. Otras intervenciones sociales, en el campo asistencial, están siendo eliminadas o reformuladas de manera progresiva y capilar, sin alcanzar una visibilidad pública tan significativa, entre ellas las relativas a la promoción de la economía social.

Esta breve caracterización de la relación entre seguridad social y asistencia contribuye a comprender la desactivación de la estrategia de inclusión por el trabajo que caracterizó al kirchnerismo como ciclo político, marcando el inicio de un proceso de reconstrucción neoliberal.

\section{A cada cual según su aporte: las reformas en el campo de la previsión social}

Durante los dos primeros años del nuevo gobierno de la Alianza Cambiemos, se sancionaron dos leyes que reconfiguraron el sistema previsional: la Ley 27.260 (2016) de "Reparación Histórica", en junio de 2016, y la Ley 27.426 (2017) de "Reforma Previsional", aprobada en diciembre de 2017. Nuestra hipótesis es que uno de los núcleos de estas transformaciones estuvo dado por la desarticulación de la moratoria previsional del ciclo de políticas previo, por lo que centraremos el análisis en ese punto ${ }^{6}$ y realizaremos una breve reconstrucción de los fundamentos de la moratoria.

En el proceso de reelaboración de las orientaciones relativas a la seguridad social durante el período 2003-2015, uno de los núcleos de confrontación con el pensamiento neoliberal residió en el sentido mismo de la protección. Ésta fue definida como la condición de aseguramiento frente a las contingencias de la vida del trabajador, cuestionándose la afronta hacia toda idea de buena dependencia ${ }^{7}$. Los problemas de precariedad e informalidad laboral tensionaron la centralidad asignada al empleo protegido como vía de acceso a la protección.

La moratoria previsional ${ }^{8}$ fue uno de los mecanismos que dieron respuesta al dilema respecto de los principios sobre los cuales extender las protecciones, que constituía un objetivo de gobierno9. Ella ejerció una reinterpretación extraordinaria sobre el principio contributivo, a fin de readecuarlo a la capacidad de contribución de los grupos de mayor vulnerabilidad sociolaboral y, así, reafirmar su vigencia. Aunque los parámetros y condiciones exigidas por el sistema previsional no fueron sometidos a revisión, la flexibilización del principio contributivo tuvo por efecto la incorporación al sistema de sujetos cuyas trayectorias y condiciones laborales no se correspondían con aquellas para las que el sistema había sido ideado (Danani, 2017a). La cobertura se amplió de forma inmediata: hacia 2011, un 38,4\% del total de los jubilados había accedido al beneficio vía moratoria (Ministerio de Trabajo, Empleo y Seguridad Social, 2014), en su mayoría eran mujeres y provenían del primer y segundo quintil de ingresos (Danani \& Hintze, 2013). En 2015 se llegaba a alrededor de un 95\% de cobertura de los adultos mayores (Comité Nacional MOST, 2015).

La flexibilización del principio contributivo reinscribió la protección de los trabajadores precarios e informales en el registro del trabajo, a contrapelo de las estrategias de lucha contra la pobreza dominantes en los años previos y de otras propuestas en ese entonces en circulación que la comprendían en un registro eminentemente asistencial ${ }^{10}$. Su fundamento era el reconocimiento del trabajo precario e informal como fuente de derechos, a partir de lo cual dichos colectivos pasaron a ser definidos e interpelados como trabajadores y, en virtud de esa condición, sujetos legítimos de protección. La ampliación del trabajo reconocido se basaba en un doble cuestionamiento hacia la mirada neoliberal sobre los problemas sociolaborales: se problematizó la autonomización de los problemas de empleo y de pobreza; y se puso en discusión la idea de que la informalidad laboral se debía a la voluntad de los trabajadores de evadir una legislación gravosa, visibilizando los comportamientos evasores de los empresarios ${ }^{11}$. Estos sentidos sostenían la pretensión de equiparación entre trabajadores formales e informales. 
La moratoria se complementó con una política de jerarquización del haber mínimo, segmento en el que prioritariamente se incorporaron los jubilados por moratoria (Torres Minoldo \& Sazatornil, 2014). Esto generó un efecto progresivo de achatamiento de la pirámide previsional (Danani \& Hintze, 2013), en reacción a lo cual se sucedió una ola de juicios previsionales que demandaban la aplicación de porcentajes similares de aumento a titulares con haberes superiores. Aunque en 2008 se adoptó una fórmula de incremento bianual para todos los segmentos de haber (Ley 26.417, 2008), el conflicto no fue suturado. Evidentemente, el reconocimiento hacia los colectivos precarios, informales y desocupados, y la pretensión de igualación siguieron siendo objeto de controversia.

A inicios del año 2016, transcurridos seis meses de gobierno, la Alianza Cambiemos impulsó el debate sobre el estado del sistema previsional, enfocando dos problemas sobre su capacidad protectoria. Según declaraciones públicas del presidente Macri ("Mauricio Macri realizó anuncios," 2016) y del responsable de la ANSES, Emilio Basavilbaso ("Basavilbaso: El kirchnerismo," 2016), estos eran: el cúmulo de juicios previsionales sin sentencia; y la situación de los adultos mayores sin aportes suficientes para jubilarse. El término de reparación que intituló la primera ley sancionada sintetiza el cuestionamiento hacia la justicia y conveniencia de las medidas adoptadas durante el ciclo político antecedente.

La sanción de la ley declaró la "emergencia en litigiosidad previsional" 12 y estableció una serie de mecanismos para establecer acuerdos entre los querellantes y el gobierno, a fin de dar curso al aumento retroactivo de los haberes. Esta propuesta daba legitimidad a los reclamos individuales surgidos durante el ciclo previo, respaldando una interpretación individualista del principio contributivo, según la cual los aportes conforman una propiedad personal que precede al principio de solidaridad del sistema. Funcionarios y políticos oficialistas trazaron una imagen utópica de correspondencia unívoca entre aportes y retribuciones, ocultando la diversidad de fuentes de financiamiento que sostienen el sistema (Administración Nacional de La Seguridad Social, 2011).

La Ley 27.260 (2016) también dispuso el cierre de la

La nueva estrategia en el

contexto de reconstrucción

neoliberal no pretende eliminar

la intervención social, sino

desarticular los sentidos

ligados al trabajo y a los

derechos como fundamentos de

un nuevo modelo de Estado.

\section{Por ello, las transferencias de}

ingresos continúan pero con

una nueva modalidad

individualizante $\mathbf{y}$

asistencializada. moratoria previsional que se encontraba vigente y la sustituyó por la Pensión Universal para el Adulto Mayor (PUAM). En virtud de su carácter extraordinario, se argumentaba en el debate parlamentario y en los discursos oficiales que la moratoria había sido un "parche"13 creado por el gobierno anterior, que no había resuelto de forma sostenible la imposibilidad de acceso a la jubilación de una proporción significativa de la población activa. La PUAM ponía en acto el abandono de la pretensión de equiparación entre trabajadores formales e informales, a través de la propuesta (otrora rechazada) de establecer un pilar no contributivo y asistencializado, a la manera de una red de seguridad básica, lo que desde inicios de siglo proponen diversos organismos internacionales (Lijterman, 2018). La edad para acceder a ella es mayor en comparación a las jubilaciones en el caso de las mujeres ${ }^{14}$, y la prestación corresponde a solo el $80 \%$ del haber mínimo. Su percepción no impide la continuidad de actividades laborales, con el supuesto objetivo de reunir los aportes necesarios para acceder a una jubilación ordinaria $^{15}$ : así, se cristaliza la inferioridad de la PUAM respecto del régimen contributivo en los discursos oficiales.

En conjunto, las medidas contenidas en esta primera ley se dirigieron a reestablecer un principio que, según los discursos oficiales, había sido alterado durante el kirchnerismo de manera discrecional: a cada quien según su aporte. La necesidad de ocuparse de las situaciones de vulnerabilidad no fue puesta en cuestión, pero sí el reconocimiento de derechos sociales asociado a estas acciones. La Ley 27.260 (2016) disponía que los recursos para su implementación fueran obtenidos de la aplicación de un "régimen de sinceramiento fiscal" que alentaba la declaración de bienes y capitales, eximiéndolos de explicitar el origen de los mismos y de sanciones por la evasión impositiva ${ }^{16}$. Esta cuestión monopolizó el debate parlamentario y público, mientras que la resolución de los juicios previsionales y la PUAM generaron consensos más allá del oficialismo.

La Ley de Reforma Previsional (Ley 27.426, 2007), de diciembre de 2017, completó este giro. Esta vez, los apoyos se redujeron notablemente, siendo aprobada por una mínima diferencia mientras se desarrollaban jornadas intensas de protesta social, reprimidas con violencia por las fuerzas de seguridad. Los discursos oficiales dejaron de lado la idea de reparación y se centraron en el problema fiscal del sistema previsional, 
subordinando las cuestiones relativas a la protección. La propuesta se dirigía a establecer una nueva fórmula para actualizar los haberes que, según se difundió extensivamente en la opinión pública, posibilitaría un notable ahorro fiscal ${ }^{17}$. El argumento central señalaba que, de no modificarse la fórmula heredada, podría producirse un colapso financiero del sistema (Cámara de Diputados de La Nación, 2017; Paladini, 2018).

Además, se desplegaba un cuestionamiento político que remarcaba las injusticias en las que habían incurrido las intervenciones del ciclo previo. El proyecto oficialista retomaba una demanda social sumamente conflictiva durante los gobiernos de Cristina Fernández: el establecimiento de tasas de sustitución del 82\%, móvil según la evolución de los salarios de referencia. Aunque hasta entonces los haberes se encontraban en una tasa cercana a dicho porcentaje, la propuesta oficial incluyó este punto en la Ley 27.426 (2017) definiéndolo como un "acto de justicia indudable" ${ }^{18}$. Su aplicación excluyó a los jubilados por moratoria, revirtiendo los procesos de igualación material y simbólica que habían tenido lugar e inaugurando nuevas distinciones con los sujetos adscriptos a la $\mathrm{PUAM}^{19}$. La reversión de la pretensión de equiparación se fundó tanto en un principio de justicia que jerarquizaba los aportes individuales como criterio de distribución, como en la reactivación del problema de los desincentivos a la formalidad asociado a las protecciones. Así lo argumentaba un diputado oficialista: “¿quién puede tener hoy en la Argentina un incentivo a hacer un aporte solidario al sistema previsional . . . si sabe que el gobierno le va a regalar una jubilación para ganarse un voto?" (Cámara de Diputados de La Nación, 2017). De este modo, se ejerció un notable "des"conocimiento de las redes de causalidad del trabajo informal y precario y de su asociación a derechos sociales, restringiendo los modos de reconocimiento hacia su sujeto.

\section{De la promoción de cooperativas a las transferencias de ingresos condicionadas}

En febrero de 2018 el gobierno nacional anunció el cierre de los programas de fomento de cooperativas de mayor alcance del país: Ingreso Social con Trabajo Argentina Trabaja y Ellas Hacen ${ }^{20}$. En su reemplazo, anunció la creación de Hacemos Futuro, una transferencia de ingresos condicionada para "personas en estado de vulnerabilidad que comprende la percepción de un subsidio para formación de carácter personal que facilite el acceso y permanencia en los cursos y prácticas de terminalidad educativa y formación integral" (Resolución n. 151, 2018).

Si bien el monto del nuevo subsidio mantiene el mismo valor que el otorgado a modo de retribución por el trabajo realizado en las cooperativas creadas por los mencionados programas ${ }^{21}$, la nueva estrategia de intervención enfatiza el carácter individual de la prestación y elimina definitivamente la figura de la cooperativa como núcleo de las tareas de los destinatarios.

Desde el inicio del Gobierno de Cambiemos, las cooperativas han sido blanco de denuncias y campañas activas de desprestigio por parte de los propios funcionarios públicos, con una importante repercusión en los medios de comunicación dominantes (Hopp, 2017a). Este proceso de deslegitimación recayó especialmente en aquellas cooperativas creadas en el marco de programas sociales, a partir de una interpretación sesgada que las considera causantes de una desnaturalización del cooperativismo ${ }^{22}$. En este contexto se vienen implementando cambios normativos tendientes a enfatizar objetivos de formación laboral y fortalecimiento de la empleabilidad y a quitar la participación en cooperativas como requisito de acceso y permanencia en los programas (Resolución n. 2055/2016, 2016c; Resolución n. 456, 2016a; Resolución n. 592, 2016b). Estas modificaciones fueron acompañadas de operativos territoriales de "desarme de las cooperativas" (Hopp, 2017b, p. 26) y de crecientes controles formales y burocráticos sobre su funcionamiento. Todo ello, desembocó en el cierre definitivo de los programas y seguramente de muchas de las cooperativas creadas, aunque no contamos con información oficial al respecto ${ }^{23}$.

Para continuar percibiendo la prestación, los titulares de Argentina Trabaja y Ellas Hacen, deben reinscribirse en el nuevo Hacemos Futuro, actualizar sus datos personales, y completar una encuesta referida a sus trayectorias educativas, laborales, de formación, salud, vivienda y grupo familiar. Cuatrimestralmente también deben certificar el cumplimiento de la escolaridad o capacitación requeridas como contraprestación, ante la ANSES. Frente a este cambio en la modalidad de intervención, esta agencia estatal que previamente no tenía ninguna injerencia en estos programas, comenzó a funcionar como organismo de registro y control, reforzando una mirada tecnocrática y de sospecha sobre los destinatarios. La construcción de la población objetivo de esta nueva política opera sobre el supuesto de que quienes perciben el subsidio no tienen motivación ni voluntad propia para insertarse en el mercado de trabajo, estudiar y así mejorar sus condiciones de vida, por ello se requiere el reforzamiento del control sobre el cumplimiento de las condicionalidades. De ese modo, se desconocen las causas estructurales de los problemas sociales, asociando la desocupación a un problema inscripto prioritariamente en la empleabilidad como condición individualizada ${ }^{24}$.

Para comprender la transformación actual en los fundamentos que orientan la política social destinada a poblaciones marginadas del mercado de empleo, señalaremos las potencialidades del trabajo cooperativo como 
estrategia de inclusión social. Esta breve caracterización del ciclo kirchnerista nos permitirá analizar qué implicancias concretas tiene el pasaje hacia formas de asistencia individualizada del desempleo mediante transferencias de ingresos condicionadas.

Ante los obstáculos identificados para la inserción en el mercado laboral de ciertos grupos sociales, durante el período 2003-2015, las cooperativas fueron una apuesta institucional por recuperar y poner en juego principios y valores del cooperativismo en la construcción de alternativas de empleo protegido. Los programas Argentina Trabaja y Ellas Hacen brindaban acceso a seguros de salud y permitían contabilizar años de aportes en el sistema previsional ${ }^{25}$.

Asimismo, se buscó responder de manera colectivaa problemáticas en los barrios, tales como la recolección de basura, la construcción de veredas, reciclaje, mantenimiento de edificios y espacios públicos, entre otras (Resolución n. 2476, 2010; Resolución n. 3182, 2009). La línea Ellas Hacen incorporaba, además, una perspectiva de género que priorizaba a mujeres con más de tres hijos/as o que fueran víctimas de violencia machista, brindándoles apoyo, contención y herramientas para afrontar con mayor autonomía su situación (Resolución n. 2176/13, 2013).

Las experiencias laborales, organizativas y de formación que se desplegaron en estos programas fueron muy diversas: en un extremo encontramos la experiencia que definimos como militancia cooperativista, en la que esta forma de organización del trabajo se consideraba tanto una alternativa laboral genuina como parte de un proyecto de vida colectivo. En el otro, la experiencia de asociatividad forzada o instrumental, en la que la intervención de la política social no logró construir formas de organización del trabajo genuinamente cooperativas ni dejar instalada la idea de una salida conjunta frente a los problemas de empleo una vez concluida la intervención estatal en cuestión (Hopp, 2015). En los casos en los que los destinatarios lograron constituirse como un grupo de trabajo, sus integrantes demostraron que cuando se construyen lazos solidarios es posible resolver las necesidades compartidas a través de la autogestión, entendida como una alternativa de autonomía posible.

Estas experiencias se asientan en una larga historia del cooperativismo que muestra que la autogestión del trabajo y la asociatividad son eficientes en distintas dimensiones: a) en lo económico, construyen relaciones de producción más igualitarias, que privilegian el trabajo y la satisfacción de necesidades sociales sobre el lucro individual; b) en lo social, se trata de un proceso capaz de impulsar acciones y obtener resultados que por su arraigo local, favorecen tanto a quienes participan de ella, como a las comunidades en donde se desarrollan (Coraggio, 2003); c) en lo político, se vinculan a la construcción de sistemas de representación y de toma de decisión colectiva (Dal Ri \& Vieitez, 2009); d) en lo técnico, la autogestión contribuye a la construcción de otra forma de organización y de división del trabajo eficiente y factible. Esta modalidad valora las capacidades humanas por sobre las exigencias del mercado laboral (Albuquerque, 2004; Singer, 2007).

Mediante la mencionada estrategia de deslegitimación de las cooperativas y de la estigmatización de los destinatarios, el pasaje hacia las transferencias condicionadas de ingresos que propone el nuevo Programa Hacemos Futuro, implica la ruptura de los espacios colectivos de trabajo creados y de la solidaridad recreada en los territorios en los que las cooperativas desarrollaban sus tareas cotidianas para resolver problemas comunes. En este sentido, los cambios que comenzaron a esbozarse en las normativas al inicio del ciclo de reconstrucción neoliberal y que hoy se asientan en la clausura de la principal línea de política social que representó la estrategia de inclusión por el trabajo del kirchnerismo, parecen apuntar a debilitar la organización colectiva y la movilización popular que disputa por inscribir el apoyo estatal en una perspectiva de trabajo con derechos, en contraposición a la asistencia individualizada.

Otra cuestión que observamos ante este cambio tiene que ver con las dificultades que implica lidiar con el incremento de trámites burocráticos que deben enfrentar los destinatarios que demandan tiempo, saberes y recursos que no responden a una lógica de accesibilidad y promoción de derechos de la población destinataria. Sumado a ello, en algunas localidades se ha expresado una preocupación por la inexistencia o insuficiencia de lugares habilitados por el gobierno nacional en donde inscribirse para completar los estudios y cumplir las condicionalidades que requiere el Programa ("Florencio Varela: el gobierno," 2018). Desde una perspectiva de género, podemos preguntarnos cuáles serán los mecanismos previstos para contribuir a la escolarización y capacitación de las destinatarias, sin que las dificultades para el cumplimiento de la nueva forma de contraprestación, asociadas a la situación de las mujeres responsables del hogar y del cuidado de sus hijos/as, no redunde en una doble penalización, dejándolas fuera del sistema educativo y sin un apoyo económico fundamental para el sostenimiento de su economía familiar. El proceso de individualización que supone la nueva lógica del programa, pone en riesgo tanto el trabajo colectivo como los espacios de contención, información, acompañamiento y autonomía que intentaba construir la línea Ellas Hacen.

La eliminación de las cooperativas como forma de organización de las tareas, no sólo desconoce las potencialidades de los principios del cooperativismo para resolver problemas sociales y económicos, sino que redefine el valor social del trabajo como fundamento de la integración social y limita potencialmente el acceso a la seguridad social, dando vía libre a las reglas de competencia, individualismo y discriminación del mercado. 


\section{Reflexiones finales: de la inclusión por el trabajo a la asistencia individualizada}

El objetivo del artículo fue analizar los procesos de reorganización de la protección social en Argentina en el contexto de reconstrucción neoliberal, en los que los sectores de política social analizados asumieron una intensa gravitación. En el período 2003-2015, la relación entre seguridad social y asistencia había sido tensionada, en virtud del reconocimiento de derechos sociales de grupos excluidos del empleo formal y de la pretensión de extender protecciones centradas en la figura del trabajo. En el ciclo actual, se evidencian procesos de clara reversión y clausura de aquellas transformaciones. Más allá del espíritu refundador del discurso oficial, centrado en el problema del déficit fiscal, la reestructuración impulsada en el esquema de política social no busca tanto la sostenibilidad económica de las intervenciones como socavar los fundamentos que orientaron la construcción estatal del acceso a la protección social y los modos de reconocimiento de los sujetos del trabajo instituidos durante el kirchnerismo.

En cuanto a la previsión social, hemos mostrado que un núcleo fundamental de las contrarreformas se organizó en torno a la reversión de los fundamentos en que se había sustentado la moratoria previsional y de sus efectos más virtuosos. Al respecto, se destaca el abandono de la pretensión de equiparación entre trabajadores formales e informales, justificada en una renovada gravitación de la interpretación neoliberal sobre la informalidad laboral y sobre el principio contributivo, bajo la cual se destaca la propiedad personal e individual de los aportes previsionales. Esto rompe con la lógica propia de un sistema público, solidario y de reparto. En estos discursos, el trabajo legítimo emerge como aquel que permite a los sujetos contribuir, mientras que las protecciones justas se sustentan en la retribución respecto del aporte realizado. De este modo se debilita el horizonte de igualación social y reconocimiento de derechos de poblaciones históricamente excluidas de la seguridad social, trazado en la estrategia de políticas de inclusión por el trabajo previa.

En relación con los programas dirigidos a impulsar el trabajo cooperativo, su clausura da cuenta de la alteración de las explicaciones sobre el desempleo y las consiguientes perspectivas de la integración social y económica de sus destinatarios. En este sentido, mostramos el ascenso de un fuerte cuestionamiento hacia el cooperativismo como alternativa laboral y el desconocimiento de las formas de solidaridad orientadas a la resolución de problemas comunitarios que esta forma de organización del trabajo pone en juego. La desarticulación de las cooperativas entraña la ruptura del anclaje colectivo de las intervenciones, así como el viraje hacia una explicación eminentemente individual de la situación de vulnerabilidad social, desempleo y pobreza. Como resultado de estas transformaciones, emerge un ideal de trabajo cuya legitimidad se entiende como resultado de una actividad que debe ganarse en el mercado.

Los analizadores seleccionados nos permitieron observar los rasgos y efectos generales de la nueva estrategia de política social y las particularidades que asumieron las contrarreformas en cada sector. En ambos casos, si bien la referencia al trabajo continúa presente, se refuerza un nuevo sentido ligado casi exclusivamente a la esfera mercantil. Desde esta perspectiva, el trabajo no funciona ya como fundamento de un proceso de ampliación de derechos, sino como un requisito de acceso a la protección social, evocado como un factor que justifica el merecimiento: la ayuda estatal es legítima para quien contribuye. La nueva estrategia en el contexto de reconstrucción neoliberal no pretende eliminar la intervención social, sino desarticular los sentidos ligados al trabajo y a los derechos como fundamentos de un nuevo modelo de Estado. Por ello, las transferencias de ingresos continúan pero con una nueva modalidad individualizante y asistencializada. Se trata de ayudas directas al necesitado o careciente, sin la institución de derechos, en el caso de la Pensión Universal para el Adulto Mayor, y sin enmarcarlo en procesos colectivos de trabajo protegido, en el nuevo Programa Hacemos Futuro. Se reestablecen así los límites entre empleo y seguridad social (contributiva), y asistencia social (no contributiva) al pobre o inempleable, con la estigmatización que esto supone.

\section{Referencias}

Administración Nacional de la Seguridad Social. (2011). La inclusión social como transformación: Políticas públicas para todos. Recuperado de http://observatorio.anses.gob.ar/archivos/documentos/La\%20inclusi\%C3\%B3n\%20social\%20como\%20transformaci\%C3\%B3n_Cuadernillo.pdf Administración Nacional de la Seguridad Social. (2015). Libro blanco de gestión. Buenos Aires, Argentina: Autor.

Albuquerque, P. P. (2004). Autogestión. In A. D. Cattani (Org.), La otra economía (pp. 39-46). Recuperado de https:// www.economiasolidaria.org/sites/default/files/Laotraeconomia.pdf

Basavilbaso: "El kirchnerismo se aprovechó de los jubilados". (2016, 28 de mayo). La Nación. Recuperado de https:// www.lanacion.com.ar/1903373-basavilbaso-el-kirchnerismo-se-aprovecho-de-los-jubilados

Cámara de Diputados de La Nación. (2017, 18 de diciembre). [Versión taquigráfica de la sesión parlamentaria del día 18 de diciembre de 2017]. Recuperado de http://www4.hcdn.gob.ar/sesionesxml/provisorias/135-24.htm

Castel, R. (1997). La metamorfosis de la cuestión social. Una crónica del salariado. Buenos Aires, Argentina: Paidós. 
Centro de Estudios Económicos y Sociales Scalabrini Ortiz. (2016, 19 de enero). Informe Económico Mensual - Enero 2016. Recuperado de http://www.ceso.com.ar/informe-economico-mensual-enero-2016

Centro de Investigación y Formación de la República Argentina. (n.d.). Una visión general sobre el sistema previsional. Recuperado de http://www.centrocifra.org.ar/docs/Una\%20vision\%20general\%20sobre\%201a\%20reforma\%20previsional.pdf

Comité Nacional MOST. (2015). Radiografía de las políticas sociales del siglo XXI: Las miradas populares. Recuperado de http:// www.desarrollosocial.gob.ar/biblioteca/radiografia-las-politicas-sociales-del-siglo-xxi-las-miradas-populares/

Consejo Nacional de Coordinación de Políticas Sociales. (2018). Planes, programas y prestaciones de la seguridad social. Recuperado de https://www.argentina.gob.ar/sites/default/files/planes_programas_sociales_y_prestaciones_de_la_seguridad_social_0_0.pdf

Coraggio, J. L. (2003, noviembre). Las políticas públicas participativas: ¿Obstáculo o requisito para el Desarrollo Local? Seminario Nacional "Fortaleciendo la relación Estado-Sociedad Civil para el Desarrollo Local", Buenos Aires, Argentina, 2. Recuperado de http://www.coraggioeconomia.org/jlc/archivos\%20para\%20descargar/ponencia\%20CENOC\%202.pdf

Cortés, R., \& Marshall, A. (1999). Estrategia económica, instituciones y negociación política en la reforma social de los '90. Desarrollo Económico, 59(154), 195-212.

Costa, I. \& Hintze, S. (2014). Capacidad protectoria de la Asignación Universal por Hijo para Protección Social: problemas y debates a cuatro años de implementación. In C. Danani \& S. Hintze (Coords.), Protecciones y desprotecciones (II): Problemas y debates de la seguridad social en Argentina (pp. 243-280). Los Polvorines, Argentina: Universidad Nacional de General Sarmiento.

Dal Ri, N. M., \& Vieitez, C.G. (2009). Trabajo asociado: Gestión democrática y cambio social. Observatorio Social de Empresas Recuperadas y Autogestión, (1), 1-10. Recuperado de http://webiigg.sociales.uba.ar/empresasrecuperadas/PDF/ DalRiyVieitezCastellano.pdf

Danani, C. (2017a). La capacidad de protección de la seguridad social en Argentina en el nivel nacional: instrumentos de políticas entre 2002 y 2015 y desafíos futuros. In L. Cortes da Costa \& A. H. Del Valle (Orgs.), A seguridade social no Brasil e na Argentina: Os direitos sociais em tempos de ajustes neoliberais (pp. 211-244). Guarapuava, Brasil: Unicentro.

Danani, C. (2017b). Políticas sociales universales: Una buena idea sin sujeto. Consideraciones sobre la pobreza y las políticas sociales. Revista Sociedad, (37), 77-94.

Danani, C., \& Grassi, E. (2008). Ni error, ni omisión. El papel de la política de Estado en la producción de las condiciones de vida y de trabajo. El caso del sistema previsional en la Argentina (1993-2008). In J. Lindenboim (Comp.), Trabajo, ingresos y políticas en Argentina. Contribuciones para pensar el siglo XXI (pp. 259-298). Buenos Aires, Argentina: Eudeba.

Danani, C., \& Hintze, S. (2013). Seguridad social y condiciones de vida. La protección social en Argentina entre 2002 y 2012. Voces en el Fénix, 4(23), 44-51. Recuperado de http://www.vocesenelfenix.com/content/seguridad-social-y-condiciones-de-vida-laprotecci\%C3\%B3n-social-en-la-argentina-entre-2002-y-201

Danani, C., \& Lindenboim, J. (2003). Trabajo, política y políticas sociales en los 90: ¿hay algo de particular en el caso argentino? In J. Lindenboim, J. \& C. Danani (Coords.), Entre el trabajo y la política. Las reformas de las políticas sociales argentinas en perspectiva comparada (pp. 253-266). Buenos Aires, Argentina: Biblos.

Dardot, P., \& Laval, C. (2013). La nueva razón del mundo. Ensayo sobre la sociedad neoliberal. Barcelona, España: Gedisa.

Decreto 1454/2005, 2005. Sistema Integrado de Jubilaciones y Pensiones. Recuperado de http://servicios.infoleg.gob.ar/infolegInternet/ anexos/110000-114999/111942/norma.htm

Florencio Varela: el gobierno nacional pone en peligro los haberes de 17 mil personas incluidas en Programas Sociales. (2018, 10 de abril). Cuatro Medios. Recuperado de http://www.cuatromedios.com.ar/articulo/florencio-varela/florencio-varela-alerta-cupos-cooperativasprograma-argentina-trabaja/20180410191127002103.html

Fraser, N. (1997). Iustitia Interrupta: Reflexiones críticas desde la posición "post-socialista”. Santafé de Bogotá, Colombia: Siglo del Hombre. Gago, V. (2014). La razón neoliberal: Economías barrocas y pragmática popular. Buenos Aires, Argentina: Tinta y Limón.

Golbert, L. (con Roca, E.). (2010). De la Sociedad de Beneficencia a los derechos sociales. Recuperado de http://www.trabajo.gov.ar/ downloads/seguridadSoc/delasociedaddebeneficenciaalos derechossociales.pdf

Grassi, E. (2004). Política y cultura en la sociedad neoliberal. La otra década infame (II). Buenos Aires, Argentina: Espacio.

Grondona, A. (2017). La Asignación Universal por Hijo y sus pasados. Reflexiones desde una Historia del Presente. In P. Arcidiácono \& C. Zibecchi (Coords.), La trama de las políticas sociales: Estados, saberes y territorios (pp. 61-82). Buenos Aires, Argentina: Biblos. Hopp, M. (2015). Identidades laborales de destinatarios del Programa Ingreso Social con Trabajo Argentina Trabaja. Trabajo y Sociedad, 24, 207-223. Recuperado de http://www.scielo.org.ar/scielo.php?script=sci_arttext\&pid=S1514-68712015000100011

Hopp, M. (2017a). El trabajo cooperativo en cuestión: Desafíos en el nuevo contexto argentino. Revista Ciencias Sociales, 93, $102-107$. Recuperado de http://www.sociales.uba.ar/wp-content/blogs.dir/219/files/2017/05/REVISTA-93-102-HOPP.pdf

Hopp, M. (2017b). Transformaciones en las políticas sociales de promoción de la Economía Social y del trabajo en la economía popular en la Argentina actual. Cartografias del Sur, 6, 19-41. Recuperado de http://cartografiasdelsur.undav.edu.ar/index.php/CdS/article/ download/86/77/

Instituto Nacional de Estadística y Censos. (2018). INDEC Informa, 23(4). Recuperado de https://www.indec.gob.ar/ftp/cuadros/ publicaciones/indecinforma/indec_informa_04_18.pdf

Kirchner, A. M. (2007). Politicas sociales en acción: La bisagra. Recuperado de http://www.desarrollosocial.gob.ar/biblioteca/labisagra/ 
Ley 24.476, 1995. Sistema Integrado de Jubilaciones y Pensiones. Recuperado de http://servicios.infoleg.gob.ar/infolegInternet/anexos/ 30000-34999/30341/texact.htm

Ley 26.417, 2008. Prestaciones Previsionales. Recuperado de http://servicios.infoleg.gob.ar/infolegInternet/anexos/145000-149999/ 145867/texact.htm

Ley 26.425, 2008. Sistema Integrado Previsional Argentino. Recuperado de http://servicios.infoleg.gob.ar/infolegInternet/anexos/145000149999/148141/texact.htm

Ley 26.565, 2009. Régimen Simplificado para Pequeños Contribuyentes. Recuperado de http://servicios.infoleg.gob.ar/infolegInternet/ anexos/160000-164999/161802/norma.htm

Ley 26.970, 2014. Sistema Integrado Previsional Argentino. Recuperado de http://servicios.infoleg.gob.ar/infolegInternet/anexos/230000234999/234847/norma.htm

Ley 27.260, 2016. Programa Nacional de Reparación Histórica para Jubilados y Pensionados. Recuperado de http://servicios.infoleg.gob.ar/ infolegInternet/anexos/260000-264999/263691/norma.htm

Ley 27.426, 2017. Reforma Previsional. Recuperado de http://servicios.infoleg.gob.ar/infolegInternet/anexos/305000-309999/305214/ norma.htm

Lijterman, E. (2018). Reformulaciones programáticas y reconfiguraciones en las formas expertas de conocer. Un análisis de la producción de las agencias internacionales de desarrollo (1995-2015). Revista e-latina, 16(63), 27-46.

Mauricio Macri realizó anuncios para jubilados y lanzó la amnistía fiscal. (2016, 27 de mayo). Infobae. Recuperado de https:// www.infobae.com/2016/05/27/1814491-mauricio-macri-realizo-anuncios-jubilados-y-lanzo-la-amnistia-fiscal/

Ministerio de Desarrollo Social. (2010). Políticas sociales del bicentenario. Un modelo nacional y popular (t. 1). Recuperado de http:/ /cdi.mecon.gov.ar/bases/docelec/az2086.pdf

Ministerio de Trabajo, Empleo y Seguridad Social. (2003). Libro blanco de la previsión social. Recuperado de http://www.oiss.org/wpcontent/uploads/2000/01/libroblanco-arg.pdf

Ministerio de Trabajo, Empleo y Seguridad Social. (2010). Trabajo y empleo en el bicentenario. Cambio en la dinámica del empleo y la protección social para la inclusión. Periodo 2003-2010. Recuperado de http://observatorio.anses.gob.ar/archivos/documentos/OBS000241\%20-\%20Trabajo\%20y\%20empleo\%20en\%20el\%20bicentenario.pdf

Ministerio de Trabajo, Empleo y Seguridad Social. (2014). Protección y seguridad social en la Argentina. Resultados de la Encuesta Nacional de Protección y Seguridad Social 2011. ENAPROSS. Recuperado de http://www.trabajo.gob.ar/downloads/estadisticas/enapross/ Libro_ENAPROSS_interior.pdf

Ministerio de Trabajo, Empleo y Seguridad Social. (2018). Reporte del trabajo registrado (Reporte de 25 de abril de 2018). Recuperado de http://www.trabajo.gob.ar/downloads/estadisticas/Reporte_trabajo_Abril_2018.pdf

Morell, A. (2002). La legitimación social de la pobreza. Barcelona, España: Antrophos.

Observatorio de las Elites Argentinas. (2016). Perfil sociológico de los miembros del gabinete inicial del presidente Mauricio Macri (Informe de Investigación n. ${ }^{\circ}$ 1). Recuperado de http://www.unsam.edu.ar/institutos/idaes/observatorio-elites-argentinas/informeN1.pdf Paladini, E. (2018, 10 de enero). Desde hoy, más de 1,3 millón de jubilados cobran la mínima con aumento. Clarín. Recuperado de https:/ /www.clarin.com/politica/hoy-millon-jubilados-cobran-minima-aumento_0_S1OiGO7VG.html

Palladino, A. (2018, 15 de marzo). Organizaciones sociales marcharon contra el cierre de programas laborales. Diario Contexto. Recuperado de http://www.diariocontexto.com.ar/2018/03/15/organizaciones-sociales-marcharon-contra-el-cierre-de-programas-laborales/ Pautassi, L., Arcidiácono, P., \& Straschnoy, M. (2013). AUHPS en Argentina. Entre la satisfacción de necesidades y el reconocimiento de derechos. Recuperado de https://repositorio.cepal.org/bitstream/handle/11362/6193/1/LCL3662_es.pdf

Quiroga, C. (2017, 21 de junio). Marcelo Collomb: "Ha habido una desnaturalización del cooperativismo". La Nación. Recuperado de https://www.lanacion.com.ar/2035725-marcelo-collomb-ha-habido-una-desnaturalizacion-del-cooperativismo

Resolución Ministerio de Desarrollo Social $n .^{\circ}$ 2476, 2010. Adecua PLAN NACIONAL DE DESARROLLO LOCAL Y ECONOMIA SOCIAL "MANOS A LA OBRA". Deroga resoluciones MDS No 1375/2004 y SPSyDH No 1023/2009. Recuperado de http:// digesto.desarrollosocial.gob.ar/2017/normaTexto.php?Id=246\&organismo=Ministerio\%20de\%20Desarrollo\%20Social

Resolución Ministerio de Desarrollo Social n. ${ }^{\circ}$ 3182, 2009. Recuperado de http://digesto.desarrollosocial.gob.ar/ normaTexto.php?Id=247\&organismo=Ministerio $\% 20 \mathrm{de} \% 20$ Desarrollo $\% 20$ Social

Resolución Ministerio de Desarrollo Social n. ${ }^{\circ} 456$, 2016 a. Recuperado de http://digesto.desarrollosocial.gob.ar/2017/ normaTexto.php?Id=1021\&organismo=Ministerio $\% 20 \mathrm{de} \% 20$ Desarrollo\%20Social

Resolución Ministerio de Desarrollo Social n. ${ }^{\circ}$ 592, 2016b. Recuperado de http://digesto.desarrollosocial.gob.ar/2017/ normaTexto.php?Id=1038\&organismo=Ministerio $\% 20 \mathrm{de} \% 20$ Desarrollo $\% 20$ Social

Resolución Secretaría de Coordinación y Monitoreo Institucional n. ${ }^{o}$ 2055/2016, 2016c. Recuperado de http:// digesto.desarrollosocial.gob.ar/2017/normaTexto.php?Id=1108\&organismo=Secretar\%EDa\%20de\%20Coordinaci\% F3n\%20y\%20Monitoreo\%20Institucional

Resolución Secretaría de Coordinación y Monitoreo Institucional n. ${ }^{\circ}$ 2176/13, 2013. Recuperado de http://digesto.desarrollosocial.gob.ar/ 2017/normaTexto.php?Id=432\&organismo=Secretar\%EDa\%20de\%20Coordinaci\%F3n\%20y\%20Monitoreo\%20Institucional Resolución Secretaría de Economía Social n. ${ }^{\circ}$ 151, 2018. Recuperado de http://digesto.desarrollosocial.gob.ar/ normaTexto.php?Id=1276\&organismo=Secretar $\% \mathrm{EDa} \% 20 \mathrm{de} \% 20$ Econom $\% \mathrm{EDa} \% 20$ Social 
Rofman, A., \& Zamora, A. (n.d.). Entrevista a Gabriel Vommaro: "Cambiemos en el Conurbano tiene un proyecto bonaerense unificado, pero le cuesta penetrar en las clases populares". Recuperado de http://observatorioconurbano.ungs.edu.ar/?p=8653

Singer, P. (2007). Economía solidaria. Un modo de producción y distribución. In J. L. Coraggio (Org.), La economía social desde la periferia. Contribuciones latinoamericanas (pp. 59-78). Buenos Aires, Argentina: Altamira.

Soldano, D., \& Andrenacci, L. (2005). Aproximación a las teorías de la política social a partir del caso argentino. In L. Andrenacci (Comp.), Problemas de politica social en la Argentina contemporánea (pp. 51-79). Buenos Aires, Argentina: Prometeo.

Stang, S. (2017, 24 de diciembre).Cómo les fue a los jubilados en el primer año de la nueva ley previsional. La Nación. Recuperado de https://www.lanacion.com.ar/2095013-las-claves-de-la-nueva-ley-de-movilidad-jubilatoria-como-sera-el-impacto-y-a-quienes-alcanzara Tomada, C. (2007). La recuperación del trabajo y de sus instituciones rectoras. Revista de Trabajo, 3(4), 77-90.

Tomada, C. (2014). Renovación de la regulación laboral en Argentina 2003-2013. Revista de Trabajo, 10(12), 72-80.

Torres Minoldo, M. S., \& Sazatornil, M. (2014). Inequidades distributivas de la previsión social contributiva. Aproximación al caso argentino. Temas Laborales, (127), 143-173.

\section{Notas}

1 Nos referimos a modalidades de intervención estatal que resultan dominantes en un determinado período, que tienden a construir un régimen de reproducción y pueden caracterizarse por su dimensión institucional y político-cultural, relativa a los fundamentos sobre los que se disputa su legitimidad (Danani, 2017a).

2 La búsqueda de restauración del empleo formal, tal como se la nominó en los discursos oficiales (Tomada, 2007, 2014), comprendió: a) la canalización institucional del conflicto salarial, re-estableciendo las instancias de negociación colectiva y el salario mínimo, vital y móvil; b) la regulación de la oferta y la demanda de empleo, a través de medidas que tenían el objetivo de conservar los puestos de trabajo registrados y fomentar su incremento; c) el fortalecimiento de la inspección laboral como herramienta para incrementar el registro del empleo; d) el desarrollo de iniciativas tendientes a la formación, reinserción laboral y la promoción del autoempleo.

3 No nos extenderemos al respecto, pero sin dudas, la Asignación Universal por Hijo constituyó una discontinuidad en el sector de la Seguridad Social e incluso al interior del ciclo de políticas sociales del kirchnerismo. Sobre este punto, remitimos a Ignacia Costa y Hintze (2014), Pautassi, Arcidiácono, y Straschnoy (2013) y Grondona (2017).

4 Se incorporaron más de 3,7 millones de nuevas jubiladas/os por la mencionada moratoria y 3,3 millones de niños/as al régimen de Asignaciones Familiares a través de la Asignación Universal por Hijo (Administración Nacional de La Seguridad Social, 2015).

5 La coalición política Cambiemos se conformó en el año 2015 para participar de las elecciones presidenciales. La misma está conformada por el Propuesta Republicana (PRO), la Coalición Cívica-ARI, la Unión Cívica Radical (UCR), entre otros. Para mayor profundidad, ver Observatorio de las Elites Argentinas (2016).

6 La reversión de la moratoria constituye un núcleo de sentido de diversas transformaciones operadas, aunque ellas no se agotan en este punto. Se produjeron otras modificaciones de suma relevancia a las que remitiremos de forma lateral: el incremento de la edad jubilatoria de manera opcional (a los 70 años); la continua pérdida de poder adquisitivo de los haberes a partir del cambio en la fórmula de actualización de los mismos (Centro de Investigación y Formación de la República Argentina, n.d.); y el incipiente desfinanciamiento de la ANSES, a partir de reformas impositivas (Stang, 2017).

7 Estos sentidos se evidencian en diversos documentos oficiales, tales como normativas, informes de gestión, libros de divulgación, por ejemplo: Ley 26.425 (2008), Kirchner (2007) y Ministerio de Trabajo, Empleo y Seguridad Social (2010, 2014).

8 El Decreto 1454/2005 (2005) estableció el funcionamiento de la moratoria previsional de la Ley 24.476 (1995) y, en 2014, se realizó una nueva moratoria establecida por la Ley 26.970 (2014).

9 Como mencionamos en la breve reconstrucción histórica del apartado anterior, se formularon otros modos de respuesta, como la Asignación Universal por Hijo, que extendieron la seguridad social desde el principio no contributivo, configurando un esquema protectorio organizado por fundamentos heterogéneos.

10 Los documentos oficiales dan cuenta de la gravitación hacia el año 2003 de la propuesta de establecer un pilar no contributivo basado en la comprobación de medios, con edades de acceso superiores a las jubilaciones ordinarias y prestaciones inferiores a las jubilaciones mínimas (Ministerio de Trabajo, Empleo y Seguridad Social, 2003).

11 Durante el período, la normativa laboral fue reformulada e incorporó formas de sanción a la evasión empresaria. Una de las más significativas fue el establecimiento, en 2014, de un registro público de empresarios evasores. En cambio, el déficit generado en el sistema previsional por la precariedad laboral fue absorbido como una deuda individual por parte de los trabajadores y por el Estado, que subsidió las cotizaciones. De este modo, convivieron formas diversas de visibilidad de la evasión empresarial, construyendo sentidos en tensión sobre la informalidad.

12 Artículo n. 2 de la Ley 27.260 (2016).

13 Expresión extraída del debate parlamentario de la Ley 27.426 (Cámara de Diputados de La Nación, 2017).

14 La edad de acceso a la PUAM es de 65 años para ambos sexos, mientras que la edad de acceso a la jubilación es de 65 años para los hombres y de 60 para las mujeres. Así, aumentó de hecho la edad de retiro de éstas últimas.

15 Según el artículo n. ${ }^{\circ} 6$ de la Ley 27.260 (2016): "el goce de la [PUAM] es compatible con el desempeño de cualquier actividad en relación de dependencia o por cuenta propia [y los aportes y contribuciones serán computados $]$. . . a los fines de poder, eventualmente, obtener un beneficio previsional de carácter contributivo". 
16 Ello incluía las deudas empresariales ante la seguridad social. Su declaración eximía a los empresarios de las sanciones previstas por Ley, incluyendo la inscripción en el registro público de evasores. Ver nota al pie n. ${ }^{\circ} 11$.

17 De cara a la opinión pública y a la negociación con otras fuerzas políticas, el gobierno anunció la aplicación de un bono compensatorio, por la pérdida de ingresos visualizada. En marzo de 2018 el aumento otorgado fue de 5,7\%, un valor que representa la mitad del incremento que se hubiera otorgado según la Ley anterior. Al respecto, ver Centro de Investigación y Formación de la República Argentina (n.d.). El cambio en la fórmula de movilidad alcanzó también al régimen de asignaciones familiares.

18 Extraído de la alocución del diputado oficialista Quetglas, durante el debate de la Ley 27.426 en la Cámara de Diputados de La Nación (2017).

19 En consonancia con esta orientación, el bono compensatorio mencionado en la nota n. ${ }^{\circ} 17$ comprendió únicamente a los jubilados con haber mínimo con 30 años de aporte, excluyendo a quienes accedieron al sistema vía moratoria.

20 En conjunto las líneas Programa Ingreso Social con Trabajo-Argentina Trabaja y Ellas Hacen alcanzan a 504.370 titulares. El dato corresponde al cuarto trimestre de 2017 (Consejo Nacional de Coordinación de Políticas Sociales, 2018).

21 El monto de la prestación es de 4.340 pesos que en mayo de 2018 equivalían a 177 dólares.

22 Entrevista a Marcelo Collomb, presidente del Instituto Nacional de Asociativismo y Economía Social (INAES) (Quiroga, 2017).

23 Hasta el 6 de julio de 2018, las cooperativas y mutuales de todo el país deben actualizar sus datos ante el INAES. A partir de información que ofrecen medios de comunicación alternativos, se pueden rastrear protestas por parte de las organizaciones de cooperativas creadas en el marco de los programas que se fortalecieron y se integraron a movimientos de trabajadores autogestionados existentes, a partir de la estrategia de inclusión por el trabajo impulsada durante el kirchnerismo. Un ejemplo de ello, es la denuncia realizada por Joaquín Fernández Sancha, dirigente de la Confederación Nacional de Cooperativas de Trabajo: “Tenemos la complejidad de que el Estado está apuntando contra nosotros, nos quieren cerrar la mayor cantidad de cooperativas posibles. Sólo en febrero cerraron más de 4 mil sin notificarnos, de manera arbitraria. Muchas cooperativas siguen trabajando, pero les han quitado la matrícula" (Palladino, 2018).

24 En consonancia con la estrategia de inclusión por trabajo, durante el kirchnerismo laANSES fue la principal agencia de implementación de políticas de protección social a partir de la ampliación del sujeto de la seguridad social. Con el cambio de gestión, si bien continúa teniendo un importante protagonismo, el sentido de sus intervenciones parece marcar un giro hacia la asistencia, corriendo el eje del reconocimiento del trabajo.

25 Esto se realizaba a través de la inscripción en el monotributo social, un régimen tributario subsidiado destinado a emprendedores o cooperativistas de pequeña escala para formalizar la actividad económica (Ley 26.565, 2009).

\section{Malena Victoria Hopp}

malenahopp@yahoo.com.ar

Doctorado en Ciencias Sociales por la Facultad de Ciencias Sociales de la Universidad de Buenos Aires (UBA)

Investigadora del Centro Cultural de Cooperación Floreal Gorini (CCC)

Docente de la Facultad de Ciencias Sociales en la Carrera de Trabajo Social y Investigadora del Consejo Nacional de Investigaciones Científicas y Técnicas en el Instituto de Investigaciones Gino Germani de la Universidad de Buenos Aires (UAB)

\section{UBA}

Instituto de Investigaciones Gino Germani

Pres. José Evaristo Uriburu, 950, 6to Piso - Recoleta

Ciudad Autónoma de Buenos Aires - Argentina

CPA: 1114AAD

\section{Eliana Lijterman}

eliana.lijterman@gmail.com

Máster en Investigación en Ciencias Sociales por la Facultad de Ciencias Sociales de la Universidad de Buenos Aires (UBA)

Docente de la Facultad de Ciencias Sociales en la Carrera de Trabajo Social de la Universidad de Buenos Aires (UBA)

\section{UBA}

Facultad de Ciencias Sociales

Santiago del Estero, 1029 - Constitución

Ciudad Autónoma de Buenos Aires - Argentina

CPA: C1075AAU 


\section{Agencia financiadora}

Universidad de Buenos Aires, Secretaría de Ciencia y Técnica. Proyecto UBACyT "Trabajo, asistencia, seguridad. Políticas sociales y modos de problematización en la Argentina de la reconstrucción neoliberal (2016-2019)."

Código: 20020170200063BA. Período de ejecución: 2018-2020.

Universidad de Buenos Aires, Secretaría de Ciencia y Técnica. Proyecto UBACyT "Política social y disputas políticoculturales. Continuidades y rupturas en los modos de problematización de las relaciones entre trabajo, condiciones de vida y política social en Argentina (2015-2020)."

Código: 20020170200274BA. Período de ejecución: 2018-2020.

\section{Contribuciones de las autoras}

El artículo se elaboró en conjunto, la contribución de ambas autoras es equivalente.

Aprobación por Comisión de Ética y consentimiento para participación

No se aplica.

\section{Consentimiento para publicación}

No se aplica.

\section{Conflicto de intereses}

No hay conflicto de intereses. 